$$
\begin{aligned}
F^{0}(1 \mathrm{~s}, 1 \mathrm{~s})^{\mathrm{SCF}} & =\frac{Z}{16 f_{4}{ }^{2}}\left[\frac{5}{8}+32 \cdot \frac{11+8 k+k^{2}}{(1+k)^{2}(3+k)^{3}} \cdot c\right. \\
+ & 2 \cdot \frac{1+5 k+48 k^{2}+5 k^{3}+k^{4}}{k^{2}(1+k)^{5}} \cdot c^{2} \\
& \left.+32 \cdot \frac{1+8 k+11 k^{2}}{k^{2}(1+k)^{2}(1+3 k)^{3}} \cdot c^{3}+\frac{5}{8 k^{5}} \cdot c^{4}\right] .
\end{aligned}
$$

Da bei Green ${ }^{23}$ die Energien (23) sowohl mit seinen Parameterwerten wie mit denjenigen nach
Löwdin schon berechnet sind (siehe Tab. 4), erübrigt sich dieser Teil des Problems. Es wurde hier auch nicht so sehr in bezug auf die tatsächliche Energieberechnung, sondern als Beispiel für die Auswertung der Slater-Integrale mit analytischen SCF-Funktionen gebracht.

Die Anregung zu dieser Veröffentlichung ist mir durch die Arbeiten von Herrn Prof. Dr. H. Schüler gegeben worden.

\title{
Der Isotopieeffekt bei der elektrolytischen Wanderung der Kupfer-Ionen in geschmolzenem Kupferchlorür
}

\author{
Von A. Lundén und E. Berne \\ Aus dem Institut für Physik und dem Institut für Kernchemie, Chalmers Technische \\ Hochschule, Göteborg \\ (Z. Naturforschg. 9 a, 684-689 [1954]; eingegangen am 20. Juni 1954)

\begin{abstract}
Durch elektrolytische Überführung wurde an der Grenze zwischen geschmolzenem
\end{abstract} \\ $\mathrm{CuCl}$ und $\mathrm{PbCl}_{2}{ }^{65} \mathrm{Cu}$ im Verhältnis $\mathrm{zu}^{63} \mathrm{Cu}$ angereichert. Die relative Differenz der elektro- \\ lytischen Wanderungsgeschwindigkeiten der Kupfer-Isotope wurde zu 0,0025 bestimmt. \\ Der daraus berechnete Masseneffekt $\mu=-0,080$ steht in Übereinstimmung mit einer \\ früher aufgestellten Regel.
}

$\mathrm{D}$ urch die Bestimmung des Unterschiedes der elektrolytischen Wanderungsgeschwindigkeiten der beiden Kupfer-Isotope in geschmolzenem Kupferchlorür sollte eine nach früheren Untersuchungen aufgestellten Regel ${ }^{1}$ für den Masseneffekt erneut kontrolliert werden.

\section{Vorversuche}

Isotopen-Überführungsversuche an $\mathrm{CuCl}$ sind etwas schwieriger durchzuführen als entsprechende Versuche an anderen Salzen, weil das $\mathrm{CuCl}$ wèder mit der Kathode noch mit der Anode in Berührung kommen sollte. Denn an der Kathode pflegen sich Kupfer-Dendrite zu bilden, und an der Anode wird das gut leitende $\mathrm{CuCl}$ in schlecht leitendes ${ }^{2} \mathrm{CuCl}_{2}$ umgewandelt. In der Tat beobachteten wir an einem Vorversuch, bei dem ein in $\mathrm{CuCl}$ eintauchendes Kupferblech als Kathode bei $530^{\circ} \mathrm{C}$ zwanzig Stunden lang mit $18 \mathrm{~mA} / \mathrm{cm}^{2}$ belastet wurde, daß neben dicht kristallisierten Kupfer-Abscheidungen ein langer, die Zelle durchziehender Kupfer-Dendrit entstanden war. Dies hatten wir an sich nicht erwartet, da sich nach Literaturangaben ${ }^{3,4}$ Dendriten

1 A. Klemm, E. Lindholm u. A. Lundén, Z. Naturforschg. 7a, 560 [1952].

${ }^{2}$ W. Biltz u. W. Klemm, Z. anorg. allg. Chem. 152, 267 [1926]. erst bei Stromdichten oberhalb $40 \mathrm{~mA} / \mathrm{cm}^{2}$ bilden sollten. Es ist uns bei unseren Vorversuchen weder durch geringe kathodische Stromdichte noch durch mechanische Maßnahmen gelungen, das Dendritenwachstum hintanzuhalten.

Umgekehrt zeigte ein Vorversuch mit $\mathrm{CuCl}$ an der Anodenkohle bei $620^{\circ} \mathrm{C}$, daß sich, offenbar infolge der Bildung von $\mathrm{CuCl}_{2}$, nur sehr geringe Elektrolyseströme auf die Dauer aufrechterhalten ließen. Deshalb wurde bei den weiteren Versuchen einerseits zwischen das $\mathrm{CuCl}$ und die Kathodenkohle $\mathrm{LiCl}$ oder besser eutektisches Gemisch von $\mathrm{LiCl}$ und $\mathrm{KCl}$ geschaltet, wobei die kathodische $\mathrm{Ab}$ scheidung von Alkali durch $\mathrm{Cl}_{2}$-Zufuhr verhindert wurde, und andererseits zwischen das $\mathrm{CuCl}$ und die Anodenkohle $\mathrm{PbCl}_{2}$.

Zunächst wurden in einer Apparatur aus Supremaxglas ähnlich der in Abb. 1 dargestellten Apparatur der Hauptversuche Vorversuche gemacht, bei denen sich kathodenseitig reines $\mathrm{LiCl}$ befand.

Das ganze äußere Rohr und das untere Stück des Diaphragmarohres war mit Bleichlorid gefüllt. Der Rest des Diaphragmarohres enthielt Kupferchlorür,

${ }^{3}$ A. H.W. Aten, H.J. den Hertog u. L. Westenberg, Trans. electrochem. Soc. 47, 265 [1925].

${ }^{4}$ W. J. Kroll, Trans. electrochem. Soc. 87, 551 [1945]. 
dessen Oberfläche etwa $1 \mathrm{~cm}$ über dem Diaphragma stand. Lithiumchlorid wurde über das Kupferchlorür geschichtet. Die Kette wurde bei $620^{\circ} \mathrm{C}$ einem Gleichstrom von $200-250 \mathrm{~mA}$ ausgesetzt, was einer Stromdichte von $4-5 \mathrm{~A} / \mathrm{cm}^{2}$ im Diaphragma, aber nur von $0,2-0,3 \mathrm{~A} / \mathrm{cm}^{2}$ an der Grenze $\mathrm{LiCl} / \mathrm{CuCl}$ entspricht.

Das geschmolzene Kupferchlorür war tiefschwarzgrün und ganz undurchsichtig, wurde aber während der Elektrolyse im Diaphragma entfärbt. Nach ein paar Stunden war die ganze Kupferchlorürsäule des Diaphragmas weiß, grau oder orangefarbig mit Ausnahme eines etwa $1 \mathrm{~cm}$ hohen schwarzen Ringes gerade an der Grenze zum Bleichlorid. (Die Farbe des Kupferchlorürs war nicht ganz dieselbe bei sämtlichen Versuchen.) Diese Beobachtung steht in guter Übereinstimmung mit der Annahme von Biltz und Klemm ${ }^{5}$, daß die schwarze Farbe des geschmolzenen Kupferchlorürs von der Anwesenheit sehr kleiner Mengen Kupferchlorid herrührt. Das schlecht leitende zweiwertige Kupferchlorid wird in unserem Diaphragma in der Richtung der Anode verdrängt und vermutlich an der Grenze $\mathrm{CuCl} / \mathrm{PbCl}_{2}$ angereichert.

Auch wenn wir am Anfang eine scharfe Grenze $\mathrm{LiCl} / \mathrm{CuCl}$ hatten, bekamen wir meistens bald Kupferdendriten an der Kathodenkohle, und diese Dendriten wuchsen durch das Diaphragma bis zum Bleichlorid, wo dann Bleimetall ausgefällt wurde. Es war sehr schwierig, diese Bildung von Dendriten zu verhindern, denn bei der verhältnismäßig hohen Temperatur $\left(620^{\circ} \mathrm{C}\right)$ und niedrigen Stromdichte $\left(0,2-0,3 \mathrm{~A} / \mathrm{cm}^{2}\right)$ an der Grenze wurde das Kupferchlorür und das Lithiumchlorid wahrscheinlich durch Diffusion z. Tl. vermischt. Der Quotient der Leitfähigkeiten von $\mathrm{LiCl}$ und $\mathrm{CuCl}$ ist 1,6, also nicht viel größer als bei $\mathrm{LiCl}$ und $\mathrm{AgCl}$ (Quotient 1,4) für welche Floberg, Klemm und Lang $^{6}$ bei der Elektrolyse einer Kette dieser beiden Salze sogar eine Diffusion im Trennrohr, wo eine große Stromdichte herrschte (etwa $6 \mathrm{~A} / \mathrm{cm}^{2}$ ), beobachtet haben. Wahrscheinlich könnten wir durch einige Kunst-

${ }^{5}$ W. Biltz u. W. Klemm, Z. physik. Chem. 110, 328 [1924]. Vgl. O. Hönigschmid u. Th. Johannsen, Z. anorg. Chem. 252, 364 [1944].

${ }^{6}$ S. Floberg, A. Klemm u. C. Lang, Z. Naturforschg. 8 a, 562 [1953].

${ }^{7}$ S. Żemcżużny u. F. Rambach, Z. anorg. allg. Chem. 65, 403 [1910].

* Die Leitfähigkeit eines Salzgemisches ist gewöhnlich nicht additiv aus der der beiden Komponenten zusammengesetzt. Vgl. z. B. H. Bloom u. E. Heymann, Proc. Roy. Soc. A 188, 392 [1947]. S. W. Karpatchew, A. G. Stromberg u. W. N. Podtchai- griffe die Dendritbildung verhindern und diese Kette für die Kupferanreicherung benutzen. Wir beschlossen aber, womöglich eine Kette mit niedrigerer Arbeitstemperatur zu verwenden.

Da es kein reines Salz gibt, durch das das hochschmelzende $\mathrm{LiCl}$ ersetzt werden könnte, senkten wir den Schmelzpunkt des Lithiumchlorides durch Vermengen mit Kaliumchlorid. Ein eutektisches Gemisch dieser beiden Salze (40,5 Mol-Proz. KCl und 59,5 Mol-Proz. LiCl) schmilzt bei $352^{\circ} \mathrm{C}^{7}$. Die Leitfähigkeit der beiden Komponenten eines solchen Salzgemisches ist von der größten Bedeutung für die Möglichkeit, eine scharfe Grenze zwischen diesem Gemisch und dem Kupferchlorür zu bekommen. Die Temperaturabhängigkeit der Leitfähigkeiten der geschmolzenen reinen Salze ist früher untersucht worden ${ }^{2}$, und durch Extrapolation kann man die Leitfähigkeit für geschmolzenes Kaliumchlorid und Lithiumchlorid bei $530^{\circ} \mathrm{C}$ näherungsweise schätzen*. Man bekommt $1,5 \Omega^{-1} \mathrm{~cm}^{-1}$ für $\mathrm{KCl}$ und $5,5 \Omega^{-1} \mathrm{~cm}^{-1}$ für LiCl. Wenn man dieses mit dem Wert 3,5 $\Omega^{-1} \mathrm{~cm}^{-1}$ für $\mathrm{CuCl}$ vergleicht, sieht man, daß man keine scharfe Grenze erwarten kann, und man kann sogar befürchten, daß das Kupferchlorür im Trennrohr zum großen Teil von Kaliumchlorid verdrängt werden würde. Unsere Versuche zeigten aber, daß dieses Verdrängen nur sehr langsam vor sich geht.

\section{Hauptversuche}

Die Kette Kathodenkohle $+\mathrm{Cl}_{2} / \mathrm{LiCl}+\mathrm{KCl} / \mathrm{CuCl} /$ $\mathrm{PbCl}_{2} /$ Anodenkohle $+\mathrm{Cl}_{2}$ wurde in der in Abb. 1 dargestellten Apparatur elektrolysiert. Wie bei früheren Anreicherungen bestand das Diaphragmarohr aus Supremaxglas, das übrige Gefäß aus Pyrexglas. Die wichtigsten Daten der beiden analysierten Hauptversuche sind in Tab. 2 angegeben.

Die durch Schmelzen in Vakuum vorbereiteten Salze wurden in Stücken in die Apparatur gefüllt. Das Bleichlorid wurde zuerst eingefüllt, und als dieses Salz geschmolzen war und es das Diaphragma fast gefüllt

nowa, Zh. Obshch. Khim. 5, 1517 [1935], haben die Leitfähigkeit von geschmolzenen KCl-LiCl Gemischen bei verschiedenen Temperaturen gemessen. Für das eutektische Gemisch ist bei $800^{\circ} \mathrm{C}$ die Leitfähigkeit etwa $30 \%$ kleiner als eine additive Zusammensetzung ergeben würde. Die Leitfähigkeit der festen Gemische von diesen beiden Salzen ist bei verschiedenen Temperaturen von A. Ben rath und H. Tesche, Z. physik. Chem. 96, 474 [1920] untersucht worden. Ihre Messungen bei $320^{\circ} \mathrm{C}$ ergeben Leitfähigkeiten, die sich fast additiv aus den Werten der beiden Komponenten zusammensetzen. 
hatte, wurde die berechnete Menge Kupferchlorür auf die obere Filterplatte des Diaphragmarohrs geschichtet. Über das geschmolzene Kupferchlorür wurde ein wenig festes Lithiumchlorid geschichtet, auf welches Stücke des eutektischen Gemisches $(\mathrm{KCl}+\mathrm{LiCl})$ gelegt wurden. Durch die Verwendung dieser Zwischenschicht von reinem Lithiumchlorid verhinderten wir ein teilweises Auflösen der zugegebenen Stücke des

\begin{tabular}{|c|c|c|c|c|c|c|}
\hline & $\begin{array}{c}\text { Schmp. } \\
{\left[{ }^{0} \mathrm{C}\right]}\end{array}$ & $\begin{array}{l}\text { Sdp. } \\
{\left[{ }^{0} \mathrm{C}\right]}\end{array}$ & {$\left[\begin{array}{c}\varrho_{620^{\circ}} \\
{\left[\mathrm{g}^{-3} \mathrm{~cm}^{-3}\right]}\end{array}\right.$} & $\begin{array}{c}x_{620^{0}} \\
{\left[\Omega^{-1}\right.} \\
\left.\mathrm{cm}^{-1}\right]\end{array}$ & {$\left[\mathrm{g} \mathrm{cm}_{530^{0}}{ }^{-3}\right]$} & $\begin{array}{c}\varkappa_{530^{0}} \\
{\left[\Omega^{-1}\right.} \\
\left.\mathrm{cm}^{-1}\right]\end{array}$ \\
\hline $\mathrm{CuCl}$ & 422 & 1366 & 3,52 & 3,71 & 3,59 & 3,49 \\
\hline $\mathrm{PbCl}_{2}$ & 501 & 950 & 4,73 & 2,01 & 4,86 & 1,59 \\
\hline $\mathrm{LiCl}^{2}$ & 613 & 1353 & 1,49 & 5,86 & - & - \\
\hline $\mathrm{KCl}$ & 776 & 1500 & - & - & - & - \\
\hline $\mathrm{LiCl}$ & & & & & & \\
\hline$+\mathrm{KCl}$ & 352 & - & - & - & - & - \\
\hline eutekt & isches & & & & & \\
\hline
\end{tabular}

Tab. 1. Eigenschaften der gebrauchten Salze.

\begin{tabular}{|c|c|c|}
\hline Versuch & A & B \\
\hline Dauer (Stunden) & 70 & 80 \\
\hline Elektrolysestrom, Mittelwert (mA) & 194 & 196 \\
\hline Transportierte Ladung (Coulomb) . & 48700 & 56500 \\
\hline Stromdichte $\left(\mathrm{A} / \mathrm{cm}^{2}\right)$. $. \quad . \quad$. & 4,1 & 3,5 \\
\hline Temperatur $\left({ }^{0} \mathrm{C}\right)$ & 535 & 530 \\
\hline Trennrohr, Innendurchmesser (mm) & 3,8 & 4,1 \\
\hline Trennrohr, Länge (mm) & 170 & 170 \\
\hline Füllpulver, Siebfraktion (DIN). & $30-40$ & $24-30$ \\
\hline Freies Trennrohrvolumen (\%) & - & 42 \\
\hline Elektrolysespannung, & & \\
\hline Mittelwert (Volt) & 54 & 49 \\
\hline
\end{tabular}

Tab. 2. Versuchsdaten der Hauptversuche.

Chloridgemisches in dem schon geschmolzenen Kupferchlorür. Nun löste sich statt dessen das feste Lithiumchlorid ziemlich langsam sowohl in Kupferchlorür als auch im KCl-LiCl-Gemisch, und wir bekamen eine ziemlich scharfe Grenze zwischen dem schwarzen Kupferchlorür und dem durchsichtigen und farblosen Alkalichloridgemisch.

Am Anfang der Elektrolyse wurde die etwas diffuse $\mathrm{CuCl} / \mathrm{PbCl}_{2}$ - Grenze im Diaphragma schärfer. Die Grenze fand bald ihre Gleichgewichtslage, und sie verblieb während des ganzen Versuches fast an derselben Stelle. Mit Ausnahme von einem schwarzen Ring an der Grenze wurde die ganze Kupferchlorürsäule des Diaphragmas weiß bis orangefarbig, gerade wie es bei den Vorversuchen beobachtet worden war. Im Kathodenraum wurde wie erwartet die Grenze $\mathrm{KCl}+\mathrm{LiCl} / \mathrm{CuCl}$ bald sehr unscharf. Trotzdem hatten wir nicht so große Schwierigkeiten mit der Dendritbildung, wie wir sie bei den bei $620^{\circ} \mathrm{C}$ durchgeführten Vorversuchen ohne Kalium- chlorid hatten. Bei der Zerlegung der Zelle nach der Erstarrung konnten wir im Kathodenraum der Zelle A einige Kupferkristalle finden, aber keine langen zusammenhängenden Dendritdrähte wie bei $620^{\circ}$.

Die Stromstärke wurde auf $200 \mathrm{~mA}$ mit einer Eisen-Wasserstoff-Stabilisatorröhre (Philips Miniwatt $\mathrm{C} 8$ ) konstant gehalten. Ein Milliampèremeter wurde in bestimmten Zeitintervallen abgelesen. Die Schwankungen des Stromes waren klein, und wir konnten mit genügender Genauigkeit die durchgegangene Ladungsmenge berechnen.

Bei der Erstarrung nach der Elektrolyse verschob sich die Grenze im Diaphragma nur um ein paar mm nach unten. Bei der Zerlegung der erkalteten Apparatur wurde das Diaphragma in Stücke von ca. $2 \mathrm{~cm}$ Länge geteilt (Abb. 1). Nach Wägen dieser

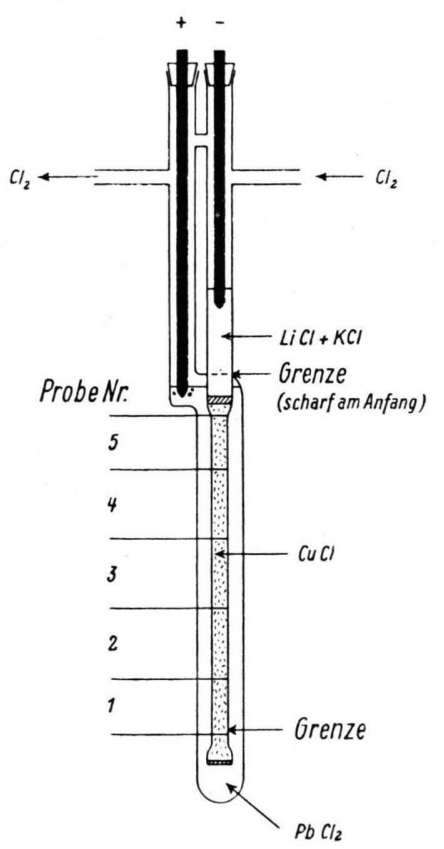

Abb. 1. Das Elektrolysiergefäß. Die Zerlegung des Trennrohres ist schematisch angegeben. Bei Versuch A liegt die Grenze $\mathrm{CuCl} / \mathrm{PbCl}_{2}$ in Probe 2, bei Versuch B in Probe 1.

Stücke wurde das Salz mit Salpetersäure herausgelöst. Durch das Wägen des Glases erhielt man das Gewicht des Salzgemisches in den einzelnen Stükken. Die verschiedenen Lösungen wurden zuerst mit Schwefelsäure versetzt, wodurch eventuell vorhandenes Bleichlorid als Bleisulfat nach Abtrennung im Filtertiegel bestimmt werden konnte. Durch Elektrolyse der verdünnten Lösungen wurde das Kupfer auf Platinelektroden ausgefällt und die vor- 
handen gewesene Menge Kupferchlorür berechnet. Die zurückgebliebene Lösung sollte nun nur Lithium und Kalium als Metallionen enthalten. Durch Eindampfen in einer Platinschale wurde ihre Summe als Sulfat bestimmt. Das Sulfat wurde aufgelöst, und das Kalium wurde als Kaliumperchlorat in gewöhnlicher Weise bestimmt ${ }^{8}$. Die entsprechenden Mengen von Kaliumchlorid und Lithiumchlorid wurden berechnet. Die Analysenergebnisse sind in Tab. 3 für Versuch A und in Tab. 4 für Versuch B angegeben. (Zuerst wurde Versuch B nach teilweise etwas abweichenden Methoden analysiert. Lithiumchlorid wurde dabei nicht und Kaliumchlorid nur unsicher bestimmt.)

\begin{tabular}{|c|l|c|c|c|c|c|}
\hline $\begin{array}{c}\text { Probe } \\
\text { Nr. }\end{array}$ & $\begin{array}{c}\text { Länge } \\
{[\mathrm{mm}]}\end{array}$ & $\begin{array}{c}\text { Salz- } \\
\text { menge } \\
{[\mathrm{mg}]}\end{array}$ & $\begin{array}{c}\mathrm{PbCl}_{2} \\
{[\mathrm{mg}]}\end{array}$ & $\begin{array}{c}\mathrm{CuCl} \\
{[\mathrm{mg}]}\end{array}$ & $\begin{array}{c}\mathrm{KCl} \\
{[\mathrm{mg}]}\end{array}$ & $\begin{array}{c}\mathrm{LiCl} \\
{[\mathrm{mg}]}\end{array}$ \\
\hline 1 & 28 & 758,2 & 754,7 & 0 & 0 & 0 \\
2 & 24,5 & 497,2 & 264,2 & 235,2 & 5,9 & 4,6 \\
3 & 32 & 608,7 & 0 & 595,1 & 24,4 & 14,9 \\
4 & 26 & 544,9 & 0 & 515,6 & 17,3 & 9,9 \\
5 & 17 & 330,9 & 0 & 317,3 & 5,4 & 10,2 \\
6 & - & 7763,0 & 0 & 6882 & 280 & 389 \\
\hline
\end{tabular}

Tab. 3. Chemische Analyse des Versuches A. Probe 6 liegt zum größten Teil über dem Diaphragmarohr.

\begin{tabular}{|c|c|c|c|c|c|}
\hline $\begin{array}{c}\text { Probe } \\
\text { Nr. }\end{array}$ & $\begin{array}{c}\text { Länge } \\
(\mathrm{mm})\end{array}$ & $\begin{array}{c}\text { Salz- } \\
\text { menge } \\
(\mathrm{mg})\end{array}$ & $\begin{array}{c}\mathrm{PbCl}_{2} \\
(\mathrm{mg})\end{array}$ & $\begin{array}{c}\mathrm{CuCl} \\
(\mathrm{mg})\end{array}$ & $\mathrm{KCl}$ \\
\hline 1 & 29 & 749,6 & 528,4 & 171,4 & \\
2 & 19 & 366,8 & 0 & 289,5 & \\
3 & 20 & 443,3 & 0 & 416,4 & \\
4 & 19 & 399,0 & 0 & 374,5 & \\
5 & 23 & 499,5 & 0 & 470,6 & $6 \%$ \\
6 & 38 & 1411,6 & 0 & $88,4 \%$ & $6,0 \%$ \\
\hline
\end{tabular}

Tab. 4. Chemische Analyse von Versuch B. Probe 6 liegt teilweise außerhalb des Trennrohres. Die KClBestimmungen der Proben $1-5$ sind nicht angegeben, da sie vielleicht unsicher sind.

Die meisten Proben enthielten etwa 95\% Kupferchlorür (Gewichtsprozent), aber die beiden Proben 1 und 2 des Versuches B ergaben nur etwa $80 \%$ Kupferchlorür, wobei alles Kupfer auf dieses Salz umgerechnet worden ist. Zum Unterschied von den anderen Proben, die hellbraun waren, waren diese zwei Proben teilweise schwarzfarbig bei der Zerlegung der Apparatur, und dieses bedeutet vielleicht, daß das Kupfer in diesen zwei Proben teilweise als Kupferchlorid vorlag.

${ }^{8}$ F.P. Treadwell u. W.T. Hall, Analytical Chemistry, Vol. II S. 272 (9th Ed. New York 1945).
Bei Versuch A, wo alle Komponenten bestimmt sind, haben wir die Meßwerte nicht auf die Summe $100 \%$ korrigiert. Die größten prozentuellen Fehler sind in den Bestimmungen von $\mathrm{KCl}$ und $\mathrm{LiCl}$ zu erwarten. Als Kontrolle der Meßgenauigkeit wurden bei Probe A: 6 drei Kupferbestimmungen an Teilen der Lösung gemacht. Das Ausgangs-Kupferchlorür wurde auch untersucht. Die Übereinstimmung mit der angegebenen Reinheit (höchstens $3 \%$ als $\mathrm{CuCl}_{2}{ }^{9}$ ) war sehr gut. Wir können die maximalen Fehler unserer Kupfer- und Bleibestimmungen auf $1 \%$ setzen. Zur Kontrolle der Methode der Alkalibestimmung wurde diese auf unser eutektisches Gemisch angewandt und ergab sehr gute UUbereinstimmung mit der durch Einwiegen bekannten Zusammensetzung.

Man bemerkt, daß in allen Proben des Versuches A immer Lithiumchlorid zusammen mit dem Kaliumchlorid vorhanden ist. Dieses war nach unseren Leitfähigkeitsüberlegungen nicht zu erwarten. Die chemische Untersuchung der beiden Proben A:6 und B:6, die teilweise außerhalb des Diaphragmas lagen, und des bei Versuch B über Probe 6 liegendem unteren Teiles des Kathodenraumes bestätigt, daß unmittelbar über dem Trennrohr die Schmelze zum größten Teil aus Kupferchlorür besteht.

Das Supremaxpulver des Trennrohres B wurde unter dem Mikroskop untersucht. Das Pulver war von der Salzschmelze nicht merkbar angegriffen.

\section{Isotopenanalyse}

Die Analyse wurde durchgeführt durch Neutronenbestrahlung der Proben und nachfolgendes Messen der spezifischen Radioaktivität des gebildeten ${ }^{64} \mathrm{Cu}$.

Jede Probe wurde durch Glühen in Kupferoxyd überführt. Um die Bestrahlungen unter wohldefinierten geometrischen Bedingungen durchführen zu können, wurde das Oxyd mit wenig Stärke als Bindemittel gemischt, und dann unter einem Druck von $5000 \mathrm{~kg} / \mathrm{cm}^{2}$ zu Tabletten gepreßt. Jede Probe wurde dann zusammen mit einer identischen Probe aus natürlichem Kupfer in einem kleinen Aluminiumbehälter im Kernreaktor in Kjeller, Norwegen, während 24 Stdn. bestrahlt. Nach der Bestrahlung wurden die Proben in verdünnter Schwefelsäure gelöst, und das Kupfer elektrolytisch auf einem Platinnetz ausgeschieden. Das Kupfer wurde gewogen und in konzentrierter Salpetersäure gelöst. Die Lösung wurde in einem Meßkolben auf $100 \mathrm{ml}$ verdünnt, und die Radioaktivität

${ }^{9}$ May \& Baker, Laboratory chemicals. 
von $10 \mathrm{ml}$ dieser Lösung in einem Flüssigkeitszählrohr gemessen.

Die Halbwertszeit der gemessenen Aktivität wurde jedesmal bestimmt und immer gleich der des Isotopes ${ }^{64} \mathrm{Cu}(12,8 \mathrm{Stdn}$.) gefunden. Bei jeder Messung wurden etwa $10^{5}$ Teilchen gezählt, so daß die statistische Unsicherheit der Radioaktivitätsmessungen $0,3 \%$ war. Für jede Probe wurden zwei vollständige Analysen durchgeführt. Die Ergebnisse sind in Tab. 5 zusammengestellt.

Für die Analysen gilt

$$
X_{\mathrm{p}}=X_{\mathrm{N}} \cdot \frac{I_{\mathrm{p}} G_{\mathrm{N}}}{I_{\mathrm{N}} G_{\mathrm{p}}},
$$

wo $X_{\mathrm{p}}=$ Gehalt der Probe an ${ }^{63} \mathrm{Cu}, X_{\mathrm{N}}=$ Gehalt des natürlichen Kupfers an ${ }^{63} \mathrm{Cu} ; I$ bzw. $G$ sind die gemessenen Aktivitäten und die Mengen von Kupfer. Die Unsicherheit in $I, \Delta I / I$, ist etwa $\pm 0,003$. Die gewogenen Kupfermengen betrugen ungefähr 100 mg, $\Delta G / G$ ist also $\pm 0,001$. Nach vorstehender Formel gilt dann

$$
\Delta X_{\mathrm{p}} / X_{\mathrm{p}}=0,008
$$

Bei der Verdünnung und Pipettierung der Lösungen wurden stets ausgewogene Meßkolben und Pipetten benutzt. Der Fehler dieser Operationen dürfte nicht größer als etwa $0,2 \%$ sein. Die Genauigkeit der ganzen Analyse sollte folglich $\pm 1 \%$ sein.

\begin{tabular}{|c|c|cc|c|}
\hline \multirow{2}{*}{ Versuch } & \multirow{2}{*}{ Probe Nr. } & \multicolumn{3}{|c|}{ Gehalt an ${ }^{63} \mathrm{Cu}$} \\
\cline { 3 - 5 } & & \multicolumn{2}{|c|}{ Gemessen } & Mittelwert \\
\hline \multirow{2}{*}{$\mathrm{A}$} & 2 & 64,5 & 65,7 & 65,1 \\
& 3 & 66,8 & 66,9 & 66,85 \\
& 4 & 67,8 & 67,8 & 67,8 \\
& 5 & 68,2 & 69,0 & 68,6 \\
\hline \multirow{2}{*}{$\mathrm{B}$} & 1 & 65,6 & 63,2 & 64,4 \\
& 2 & 66,5 & 65,5 & 66,0 \\
& 3 & 66,4 & 67,5 & 66,95 \\
& 4 & 68,9 & 68,2 & 68,55 \\
\hline \multicolumn{2}{|c|}{ Natürliches Kupfer } & \multicolumn{3}{|c}{} \\
(Tabellenwert)
\end{tabular}

Tab. 5. Isotopenanalyse der angereicherten Proben (Versuche A und B).

Wie aus Tab. 5 hervorgeht, ist aber die reelle Unsicherheit etwa $\pm 2 \%$. Das kann keinen anderen Grund haben als örtliche Schwankungen des Neutronenflusses im Kernreaktor. Daß diese Schwankungen über die kleinen Abstände, die während der Bestrahlung zwischen der Probe und der natürlichen Kupfertablette vorhanden sind, bis $1 \%$ betragen können, wurde auch festgestellt durch mehrere Messungen von zwei gleichzeitig bestrahlten Proben aus natürlichem Kupfer.

Die Genauigkeit der Aktivierungsanalyse kann also mit der zugänglichen Neutronenquelle nicht verbessert werden. Die einzige Möglichkeit, die Fehlergrenzen wesentlich zu verkleinern, wäre die, daß man eine sehr starke Ra-Be-Neutronenquelle benutzt und Probe und natürliches Kupfer nacheinander an demselben Ort bestrahlt. Um die Radioaktivität des Kupferisotopes ${ }^{64} \mathrm{Cu}$ mit hinreichender Genauigkeit messen zu können, müßte aber eine solche Quelle mehrere Gramm Radium enthalten.

$$
\text { 4. } \mu \text {-Bestimmung }
$$

Der Masseneffekt $\mu$ ist durch die Gleichung

$$
\mu=\frac{\Delta w}{w} \cdot \frac{m}{\Delta m}
$$

definiert. Die relative Differenz der Wanderungsgeschwindigkeiten $\Delta w / w$ wurde bei unseren Versuchen mittels der Gleichung

$$
\frac{\Delta w}{w}=\left(\frac{{ }^{65} N}{{ }^{65} N_{0}}-\frac{{ }^{63} N}{{ }^{63} N_{0}}\right) \frac{F N_{\mathrm{Cu}}}{L}
$$

berechnet $^{10} . N_{\mathrm{Cu}}$ ist die Gesamtmenge von $\mathrm{CuCl}$ in allen Proben, welche eine Anreicherung aufwiesen (Proben A: 2-5 und B: 1-4). $N_{\mathrm{Cu}}$ wird in Molen gemessen. ${ }^{63} N$ und ${ }^{65} N$ sind die entsprechenden Mengen der Isotope ${ }^{63} \mathrm{Cu}$ und ${ }^{65} \mathrm{Cu}$ in diesen Proben, während ${ }^{63} N_{0}$ und ${ }^{65} N_{0}$ die einer natürlichen Isotopenzusammensetzung dieser Proben entsprechenden Mengen sind. $F$ ist die Faradaysche Ladung und $L$ ist die von $\mathrm{CuCl}$ transportierte Ladungsmenge. Diese wird an der Grenze zum angereicherten Stück des Diaphragmas (zwischen Probe A:5 und A:6 bzw. B:4 und B:5) gerechnet.

Die mit Uhr und Ampèremeter gemessene Ladungsmenge $L_{\text {gem }}$ entspricht dem Ladungstransport durch $\mathrm{CuCl}, \mathrm{KCl}$ und $\mathrm{LiCl}$ zusammen, und wir müssen abschätzen, welche Anteile von den einzelnen Salzen befördert wurden. Wir nehmen mangels genauer Kenntnisse an, daß die Transportanteile der verschiedenen Salze in einem Gemisch den Leitfähigkeiten der reinen Salze und den Konzentrationen proportional sind. Für $\varkappa_{\mathrm{KCI}}$ und $\varkappa_{\mathrm{LiCl}}$ benutzen wir die auf $530^{\circ}$ extrapolierten Werte 1,52 $\Omega^{-1} \mathrm{~cm}^{-1}$ und 5,49 $\Omega^{-1} \mathrm{~cm}^{-1}$. Am Anfang dieses Versuches gab es keine Alkalichloride in unserem Kupferchlorür, während ihre Mengen in den Proben

${ }^{10}$ A. Klemm, Z. Naturforschg. 1, 252 [1946]. 
am Ende des Versuches durch die chemische Untersuchung bestimmt worden sind. Das Verhältnis der Volumina von $\mathrm{CuCl}, \mathrm{KCl}$ und $\mathrm{LiCl}$ im zeitlichen Mittel kann nun berechnet werden ${ }^{6}$. Für Versuch A bekommen wir 35:1 : 1. Unter Berücksichtigung der Leitfähigkeit ergibt sich, daß bei Versuch A nur $95 \%$ der gesamten Ladungsmenge durch $\mathrm{CuCl}$ überführt wurden.

\begin{tabular}{|c|c|c|c|c|c|c|c|}
\hline \multicolumn{6}{|c|}{ Versuch } & A & B \\
\hline${ }^{63} \mathrm{~N}$ & & & & & & & \\
\hline$\overline{{ }^{65} N_{0}}-\overline{{ }^{63} N_{0}}$ & 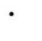 & . & & & & 0,0797 & 0,0974 \\
\hline$N_{\mathrm{Cu}}$ (Mole) & 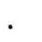 & & & & & 0,0168 & 0,0126 \\
\hline$L_{\text {gem. }}($ Coulomb) & 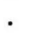 & . & & & & 48700 & 56500 \\
\hline Korrektionsfaktor & . & - & $\cdot$ & . & & 0,95 & 0,95 \\
\hline$L$ nach Korrektion & $(\mathrm{C}$ & oul & lon & ib) & & 46200 & 53700 \\
\hline$\Delta w / w$ & . & . & . & . & - & 0,0028 & 0,0022 \\
\hline Masseneffekt $(-\mu)$ & & . & . & . & . & 0,089 & 0,070 \\
\hline
\end{tabular}

Tab. 6. Die Berechnung des Masseneffektes.

In Tab. 6 sind die für die $\mu$-Berechnung wichtigen Zahlenwerte angegeben. (Für Versuch B wurde im Diaphragma das eutektische Verhältnis zwischen $\mathrm{KCl}$ und $\mathrm{LiCl}$ bei der Korrektion von $L$ angenommen.)

Als Mittelwerte der beiden Versuche bekommen wir

$$
\mu=-0,080 \text {. }
$$

Nach der früher aufgestellten Regel ${ }^{1}$

$$
\mu=-0,15\left(1+\frac{1}{2,1} \frac{m^{+}}{m^{-}}\right)^{-1}
$$

erhält man mit $m^{+}=63,6$ und $m^{-}=\mathbf{3 5 , 5}$ den Wert $\mu=-0,081$, der mit unserem Versuchsresultat in sehr guter Übereinstimmung steht. Auch bei diesem Versuch konnten wir keinen merkbaren Einfluß der Wertigkeit des Kations auf die Größe des Masseneffektes beobachten. Die Unsicherheit unserer $\mu$ Bestimmung ist aber groß, weswegen wir das Vorkommen kleinerer Abweichungen von der $\mu$-Regel nicht ausschließen können. Innerhalb der Fehlergrenzen ist die Formel für alle bisher gemessenen Salze gültig.

Diese Arbeit ist vom Schwedischen Atomkomitee finanziell unterstützt worden. Herr Professor Dr. N. Ryde hat stets für gute Arbeitsbedingungen an seinem Institut gesorgt. Herr Ziviling. S. Floberg hat bei den Versuchen selbst mitgeholfen und Herr Ziviling. R. R ajgård hat die chemischen Analysen gemacht. Wir möchten allen Beteiligten unseren herzlichen Dank aussprechen.

Der eine von uns (A. L.) hat die Schmelzelektrolyse und die Auswertung, der andere (E. B.) die Isotopenanalysen durchgeführt.

\title{
Quantitative Messungen über den Elementarprozeß der Lichtanregung von Leuchtstoffen durch einzelne $\alpha$-Teilchen. II
}

\author{
Von Immanuel Broser und Claus Reuber* \\ Aus dem Fritz-Haber-Institut der Max-Planck-Gesellschaft, Berlin-Dahlem \\ (Z. Naturforschg. 9 a, 689-693 [1954]; eingegangen am 25. Juni 1954)
}

\begin{abstract}
Die von einzelnen $\alpha$-Teilchen in Kristallphosphoren erregten Lichtblitze werden nach einem indirekten Meßverfahren untersucht. Die verwendete, neue Zählapparatur gestattet die Bestimmung von Zeitkonstante und Gesetz des Abklingvorganges. An ZnS-Cu, $\mathrm{ZnS}-\mathrm{Ag}, \mathrm{ZnS} / \mathrm{ZnSe}-\mathrm{Ag}$ werden quadratisch-hyperbolische Abklingformen, an druckzerstörtem $\mathrm{ZnS}-\mathrm{Cu}$ und $\mathrm{ZnO}$ exponentielle gefunden. Aus ersteren wird die Wahrscheinlichkeit für den mit Lichtausstrahlung verbundenen Übergang, aus letzteren die Haftstellenkonzentration bestimmt.
\end{abstract}

$\mathrm{V}$ Tor einigen Jahren berichteten Broser, Kallmann und Reuber über experimentelle Untersuchungen des zeitlichen Verlaufes der von $\alpha$ Teilchen in verschiedenen Leuchtstoffen ausgelösten Lichtblitze ${ }^{1}$. Inzwischen wurden weitere Abkling-

* Diese Arbeit enthält wesentliche Teile der Dissertation von C. Reuber, TU Berlin 1953.

${ }^{1}$ I. Broser, H. Kallmann u. C. Reuber, Z. Naturforschg. 5a, 79 [1950].

${ }^{2}$ W. Hanle, Naturwiss. 8, 176 [1951]. untersuchungen von verschiedenen Autoren durchgeführt, wobei allerdings die Anwendung der Leuchtstoffe im $\gamma$-Szintillationszähler im Vordergrund stand. (Zusammenfassende Arbeiten von $\mathrm{Hanle}^{2}$, Garlick ${ }^{3}$, Birks ${ }^{4}$ und Curran ${ }^{5}$.) Hauptsächlich

${ }^{3}$ G. F. J. Garlick, in ,,Progress in Nuclear Physics" 2, 51 [1952].

${ }^{4}$ J. B. Birks, Scintillation Counters, London 1953.

5 S. C. Curran, Luminescence and the Scintillation Counter, London 1953. 